\title{
Research and Application of Optimum Coding STMV Beamformer with Missing Sensors
}

\author{
Wang Ping ${ }^{1, \text { a }}$, Tang Ji-wei ${ }^{2}$ \\ ${ }^{1}$ Shanghai Marine Electronic Equipment Research, 201108, China \\ ${ }^{2}$ Shanghai Radio Equipment Research Institute, 200090, China \\ aheuwangping@163.com
}

Keywords: Missing sensors; sidelobe suppressions; optimum coding.

\begin{abstract}
In this paper, we propose an optimum coding STMV beamformer to improve the performance of suppressing high peak sidelobe level with missing sensors. It considers the choices of sub-array geometry have a significant effect on the performance of STMV method using sub-array (we call it STMV-SA), and the geometry of the original array is described as code, where sub-arrays processing is equivalent to the sub-coding processing. The validity and feasibility of the proposed method is tested by the theory deducinglcomputer simulations and real-data processing.
\end{abstract}

\section{Introduction}

Beamforming as one of the most important technologies, which could improves the signal processing performance and the probability of detection .In the case of Uniform Line Array (ULA), the STMV(Steered Minimum Variance) $\operatorname{method~}^{[1 \sim 9]}$ gives a precision covariance matrix estimate obtained from space data is computed in a single snapshot. Swingler combines the STMV and sub-array, and proposes the STMV-SA method ${ }^{[10 \sim 13]}$ could maintain the performance of STMV and reduce the computational load, these advantages make the high resolution beamformer application availably in engineering.

At present, there are most methods of beamforming based on ULA. In the case of missing sensors due to the design of nesting array or the faulty array, which result in high peak sidelobe level ${ }^{[14 \sim 17]}$, this problem will degrade the detection of weak signal and increase the probability of false detection.

In order to suppress the high peak sidelobe level and improve the detection reliability, this paper proposed an optimum coding STMV beamformer could solve the above problems.

\section{STMV method using sub-array}

\section{STMV method}

The basic idea of STMV method is to suppress strong interferences at non-looking directions while the gain at looking direction is kept at a constant level. In a ULA with M omni-sensors, the Fourier vector $X_{k}$ is obtained by DFT, and the pre-steered beam spectrum of frequency to the direction of $\theta$ can be expressed by

$$
Y_{k}(\theta)=D\left(\theta, f_{k}\right) X_{k}
$$

Where $k$ is the index of frequency, $D\left(\theta, f_{k}\right)=\operatorname{diag}\left(\varphi_{1}, \varphi_{2}, \cdots, \varphi_{M}\right)$ is a phase-shift operator for each sensor element, and $d$ is intercessor spacing, $c$ is the speed of acoustic propagation.

The STCM matrix is based on a covariance matrix estimate obtained from wideband data processing in a single snapshot. The STCM is given by

$$
\hat{R}_{\text {stmv }}(\theta)=\sum_{k=1}^{B} Y_{k}(\theta) Y_{k}^{H}(\theta)
$$

The adaptive weight vector of STMV is given by 


$$
\hat{w}_{s t m v}(\theta)=\frac{\hat{R}_{s t m v}^{-1}(\theta) I_{M}}{I_{M}^{H} \hat{R}_{s t m v}^{-1}(\theta) I_{M}}
$$

Wherein $I_{M}$ implies a $M \times 1$ vector of ones. The matrix inverse makes the noise correlation cancellation in order to minimize sidelobe of STMV method.

\section{STMV method using sub-array}

The STMV-SA method is familiar with STMV method. These sub-arrays are beam formed using the CBF approach, and this first stage of beamforming generates new sets of beams equal to the number of sub-arrays, and we consider the sub-arrays as the new array's elements. The second stage of the STMV on a set of beams which are steered in the same direction space but each belongs to a different sub-array. Fig 1 shows that, where a new array contains $J$ elements, and $j$ is the index of elements, such as $(j=1,2, \cdots, L)$. In order to describe conveniently, we call each sub-array's beam output is $R_{1 j}(\theta)$, and the new array's beam output is $R_{2}(\theta)$. The performance of original array's beamformer is rest with both of them.

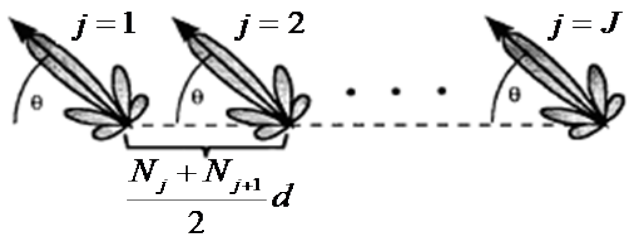

Fig.1 The configuration of sub-array to form the new array

The pre-steed frequency spectrum of the new array is by

$$
U_{k}(\theta)=K_{N} K^{T} Y_{k}(\theta)
$$

Where $K_{N}=\operatorname{diag}\left(1 / N_{1}, 1 / N_{2} \cdots, 1 / N_{J}\right)$ is a normalization matrix, and $N_{j}(j=1,2, \cdots, J)$ is the number of sensor within the $j^{\text {th }}$ sub-array. Where $K$ arranged as the columns of a $N \times J$ data matrix, in the sub-array selection matrix $K$, each row indicates a sub-array, and each row contains " 1 " and " 0 " which indicates the sensor is selected or not. In (2), substituting $U_{k}(\theta)$ for $Y_{k}(\theta)$, we can estimate the STCM of sub-array

$$
\hat{R}_{s a}(\theta)=\sum_{k=1}^{B} U_{k}(\theta) U_{k}^{H}(\theta)
$$

When $N_{1}=N_{2}=\cdots=N_{J}=1$, it is easy to know that $U_{k}(\theta)=Y_{k}(\theta)$, and STMV method using sub-array is identical to STMV method.

\section{The theory deducing of optimum coding STMV beamformer with missing sensors}

It should be consider the relation between the geometry of the sub-arrays and the index of missing sensors. The structure with missing sensors is described as " 1 " and " 0 ", which indicates the sensor is being or absent. Fig 2 is the description of missing sensors with codes.
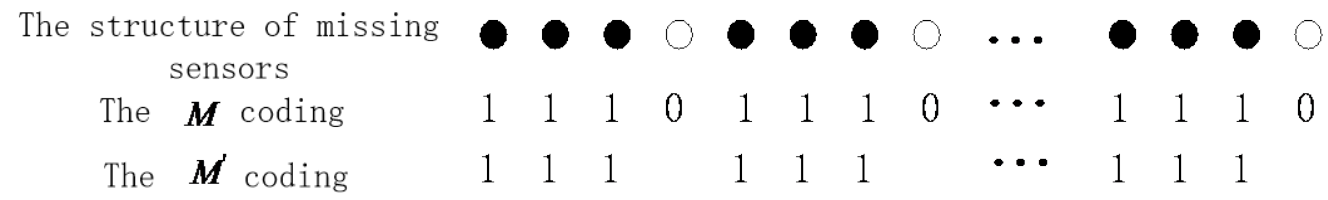

Fig. 2 The description of missing sensors with codes

The $\boldsymbol{M}$ coding is divided into $\boldsymbol{J}$ sub-codings which are equivalent the sub-arrays, the original array can be described as sub-coding ,for example, $\boldsymbol{M}=\left[\begin{array}{lllll}\boldsymbol{M}_{1} & \boldsymbol{M}_{2} & \cdots & \boldsymbol{M}_{J}\end{array}\right]$, and 
( $\left.\begin{array}{llll}j=1 & 2 & \cdots & J\end{array}\right)$, we define $l_{j}$ is the length of the $M_{j}$ sub-coding contains $j$ codes. The length of array is the sum of all the sub-codings.

$$
L_{M}=\sum_{j=1}^{J} l_{j}
$$

And the space between adjacent sub-codings is defined by

$$
d_{j, j+1}=\left(l_{j}+l_{j+1}\right) / 2 \text {, }
$$

When optimising the array it is important to have a cirteria to judge the performance of array configurations.Generally, minimising the peak sidelobe level is considered the most importatn task as this reduces the probability of false detection and the performance of optimum coding STMV is encoded with this effect. It is proved that the greater difference of sub-codings and the greater random sapce of sub-codings, which make the stronger interferences diffuse at most angle, and deserve lower sidelobe and superior performance(the conclution is proved by the following simulations). The mathetical theory could be expressed by

$$
N=N_{1}+N_{2} \rightarrow \operatorname{Max}
$$

Where,

$$
\begin{gathered}
N_{1}=J-\sum_{i, j} M_{i} \cap M_{j}, \quad(i \neq j, \text { and } i, j=1,2, \cdots, J) \\
N_{2}=(J-1)-\sum_{i, j} d_{i, i+1} \cap d_{j, j+1}, \quad(i \neq j, \text { and } i, j=1,2, \cdots, J-1)
\end{gathered}
$$

Wherein $N_{1}$ and $N_{2}$ indicate separately the number of different sub-codings and the number of different space between adjacent sub-codings. With statisfying the constrain condition of (8) and (9), we can get the optimum sub-coding. When $N=2$, it indicates all the sub-coding and the space between adjacent sub-codings is the same, and the sidelobs of beamformer will be degrated by stronger interferences.

By deleting " 0 “ of the $M$ coding we get the moidified $M$ ' coding, which contains all the elements is "1". Similarly, the former array can be described as modified sub-codings such as $M^{\prime}=\left[\begin{array}{llll}M_{1}^{\prime} & M_{2}^{\prime} & \cdots & M_{J}^{\prime}\end{array}\right]$, and $l_{j}^{\prime}$ is the length of the $M_{j}^{\prime}$ sub-coding. The pre-steed frequency spectrum of modified STMV is by

$$
Y_{k}^{\prime}(\theta)=D^{\prime}\left(\theta, f_{k}\right) X_{k}
$$

Where $\boldsymbol{D}^{\prime}\left(\theta, f_{k}\right)$ is the modified phase-shift operator for each sensor element accoring to the moidified $M^{\prime}$ coding

$$
\mathbf{D}^{\prime}\left(\theta, f_{k}\right)=\operatorname{diag}\left(\varphi_{1}, \varphi_{2}, \cdots, \varphi_{M^{\prime}}\right)
$$

The pre-steed frequency spectrum of optimum coding STMV using sub-array is expressed by

$$
U_{k}^{\prime}(\theta)=K_{N}^{\prime} K^{\prime T} Y_{k}^{\prime}(\theta)
$$

Where $K_{N}^{\prime}=\operatorname{diag}\left(1 / l_{1}^{\prime}, 1 / l_{2}^{\prime}, \cdots, 1 / l_{J}^{\prime}\right)$ is a normalization matrix, and $l_{j}^{\prime}(j=1,2, \cdots, J)$ is the number of sensor within the $j^{t h}$ sub-coding. and $K^{\prime}$ is selection matrix. Substituting $U^{\prime}(\theta)$ for $U_{k}(\theta)$, we can estimate the optimum weight with missing sensors.

\section{Computer simulations and real-data processing}

\section{Computer simulations}

We set a ULA contains 24 elements, the number of sub-array is $J=[3 、 4 、 8]$, and the detail of structure of sub-array is seemed in Table 1 . For analysis the choices of sub-array geometry have a significant effect on the performance of STMV-SA method purposes, this paper considers the following conditions: 
1、 Ignored the difference of each sub-array' beam output $R_{1 j}(\theta)$ and just considering the effect on the performance of STMV based on equal space processing and unequal space processing. It is simulations by Fig 3.

2、 Comparing the performance of STMV-SA based on different sub-arrays' geometry. It is simulations by Fig 4.

Table1. The structure of sub-arrays

\begin{tabular}{|c|c|c|c|}
\hline \multicolumn{2}{|c|}{ Sub-arrays } & The sub-array geometry & The space between \\
\hline \multirow{3}{*}{$\begin{array}{l}\text { The equal } \\
\text { processing }\end{array}$} & $J=3$ & {$[8,8,8]$} & 8 \\
\hline & $J=4$ & {$[6,6,6,6]$} & 6 \\
\hline & $J=8$ & {$[3,3,3,3,3,3,3,3]$} & 3 \\
\hline \multirow{3}{*}{$\begin{array}{c}\text { The } \\
\text { unequal } \\
\text { processing }\end{array}$} & $J=3$ & {$[5,11,8]$} & {$[8,9.5]$} \\
\hline & $J=4$ & {$[4,5,6,9]$} & {$[4.5,5.5,7.5]$} \\
\hline & $J=8$ & {$[3,2,5,3,6,2,1,2]$} & {$[2.5,3.5,4,4.5,4,1.5,1.5]$} \\
\hline
\end{tabular}

In Fig 3, the real line represent unequal processing, and the dot line represent equal processing according to the Table 1.The signals arriving from $0^{\circ}$, and the frequency of signal is the same with century frequency of receiving array. In Fig 4, we get the performance of STMV-SA with equal and unequal processing, for discuss conveniently, we call the STMV-SA with equal processing equal-STMV and call the STMV-SA with unequal processing unequal-STMV.

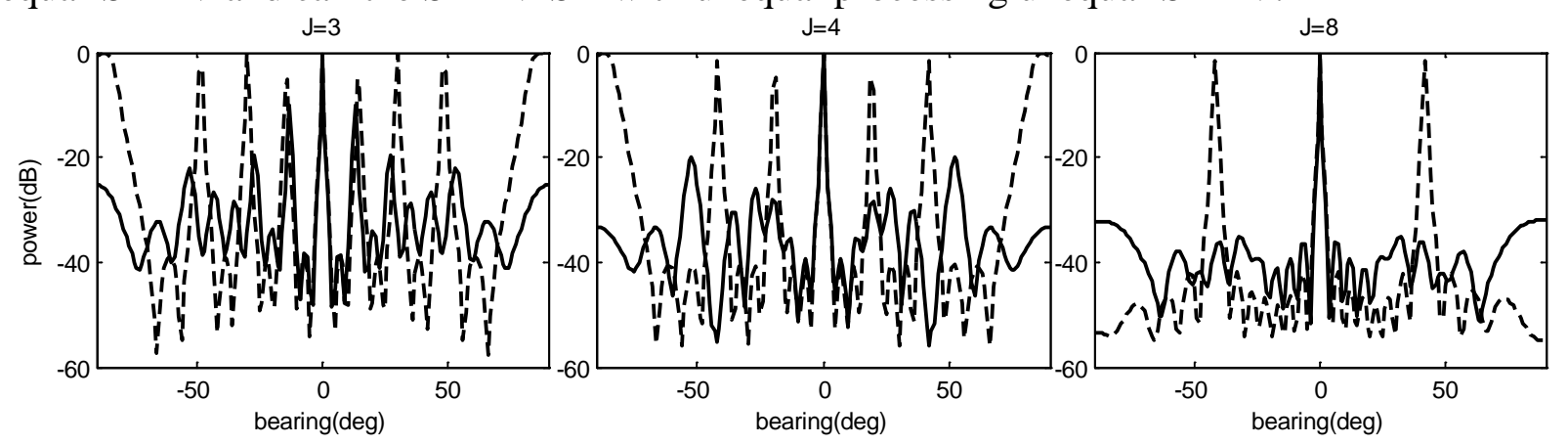

Fig.3 The normalized power spectral of STMV with equal and unequal space processing

From Fig 3, we can get the following conclusions:

(1) When the space of array exceed $1 / 2$ wavelength, the equal processing makes the $R_{2}(\theta)$ processor appeared strong interference at $\gamma$ grating lobe as if it were actually, and $\sin \gamma=\frac{\lambda_{k}}{N_{J} d} q+\sin \theta_{0}$. Where $q$ is integer, $\theta_{0}$ is the signal arriving bearing, $N_{J} d$ is the space between sub-arrays, $\lambda_{k}$ is the signal wavelength.

(2) Due to the unequal processing, the STMV can suppress the grating lobe at $\gamma$ bearing. [12]. 

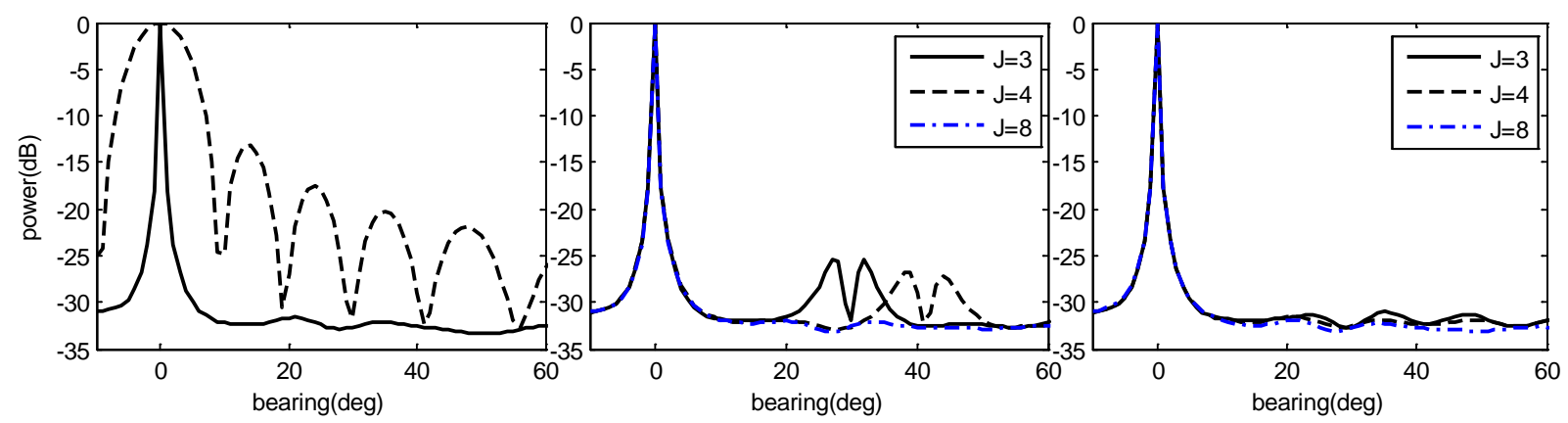

Fig.4 The normalized power spectral output

From Fig 4, we summarize the conclusions:

(1) Compared with CBF, the STMV $\backslash$ the equal-STMV and the unequal-STMV have higher robust resolution and lower peak sidelobe levels.

(2) The equal-STMV has higher peak sidelobe level at grating lobes, this is because the $R_{2}(\theta)$ processor will pass the interference, however the $R_{1}(\theta)$ can cancel the interferences by sidelobe ,and the overall effect is much lower than mainlobe, it could be seemed as sidelobe.

(3) Compared with the equal-STMV, the unequal-STMV has no obvious sidelobes contribute to the unequal processing make $R_{1 j}(\theta)$ of each sub-array is different and $R_{2}(\theta)$ get the ability to suppress the grating lobe. Computer simulations proved that with the greater difference of sub-array beam output and the greater random sapce of sub-arrays, which make the stronger interferences diffuse at most angle, and deserve lower sidelobe and superior performance.

We set a configuration of missing sensors with Fig 2, the signals arriving from $30^{\circ}$. It is supposed that $L_{M}=64$ and easy to know $L_{M^{\prime}}=48$.we deal $M^{\prime}$ code with equal sub-coding. Fig 5 plots the example of corresponding structure with $M_{j}^{\prime}=6$. Fig 6 plot the corresponding beam output for $J^{\prime}=[4,8 、 12]$ with equal $M_{j}^{\prime}$ sub-coding, and compare the performance with CBF.

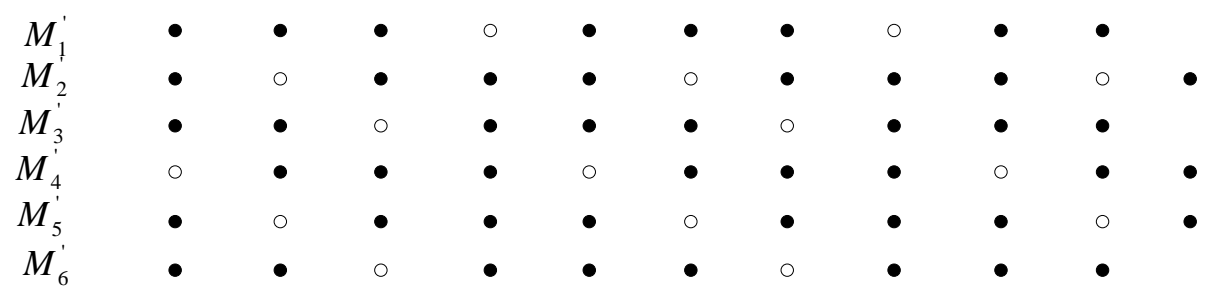

Fig.5 The structure of sub-coding with equal $M_{j}^{\prime}=6$.

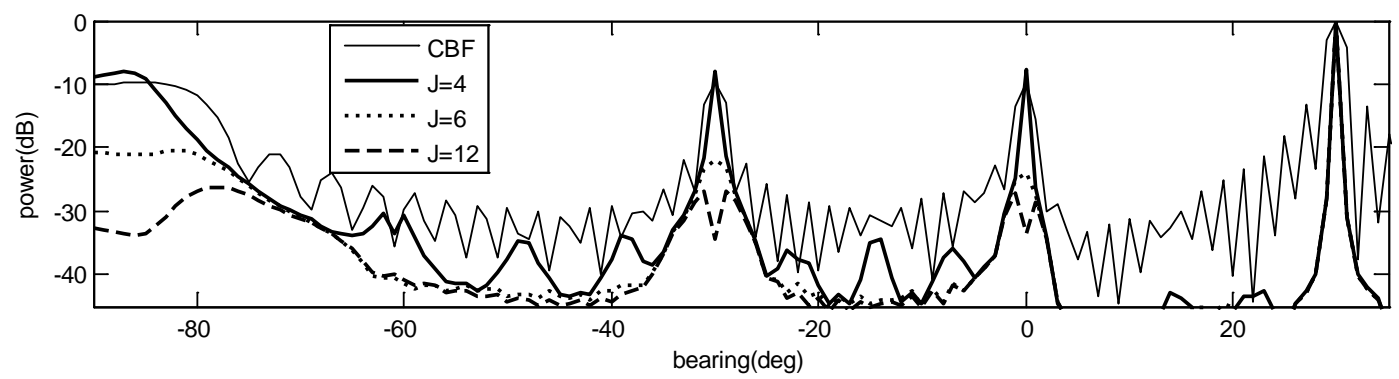

Fig.6 The normalized power spectral output

To demonstrate the performance of the proposed method, we compared the CBF method with the optimum coding STMV method using artificial data. Fig 7 demonstrates that the performance of CBF method has high sidelobe at $0^{\circ}$ 、 $-30^{\circ}$ and $-90^{\circ}$ which increasing the false alarm. When $\mathrm{J}=4$, we get $N=2$, and the equal-STMV can suppress the high sidelobes invalidate. In contrary for $\mathrm{J}=6$ or $\mathrm{J}=12$, 
it is easy to know that $N>2$, and the unequal-STMV get the superior performance. The simulations demonstrate the above conclusion, and with the $\mathrm{N}$ increases the performance is better.

Table 2 gives the statistical analysis to determine that the average sidelobe normalize power relative to the mainlobe with one hundred of Monte Carlo simulations. In general, we set $\mathrm{J}=6$ could satisfy with the most conditions of computing load of project.

Table2. The average sidelobe normalize power relative to the mainlobe

\begin{tabular}{|c|c|c|c|c|}
\hline \multirow{4}{*}{ STMV } & $\mathbf{J}$ & $\boldsymbol{l}_{\boldsymbol{i}}^{*}$ & Sidelobe level/dB & \multirow{2}{*}{ Computational load } \\
\cline { 2 - 4 } & 4 & 12 & -7.2 & \multirow{4}{*}{$\mid$} \\
& 6 & 8 & -21.5 & \\
\cline { 2 - 4 } & 12 & 4 & -26 & \\
\hline CBF & 1 & 1 & -9.6 & 1 \\
\hline
\end{tabular}

\section{Real-data processing}

Fig. 8 is the LOFAR of trial passive data, two targets have two tonal at normalized frequencies (Target A: 0.5, Target B: 0.56). The higher SNR at 0.5 normalized frequencies at $0^{\circ}$, the lower SNR at 0.56 normalized frequencies at $30^{\circ}$. We set a ULA contains 24 sensors and the configuration of missing sensors is same with Fig 2. We compare CBF with equal-STMV and unequal-STMV at the condition of $\mathrm{J}=3$.

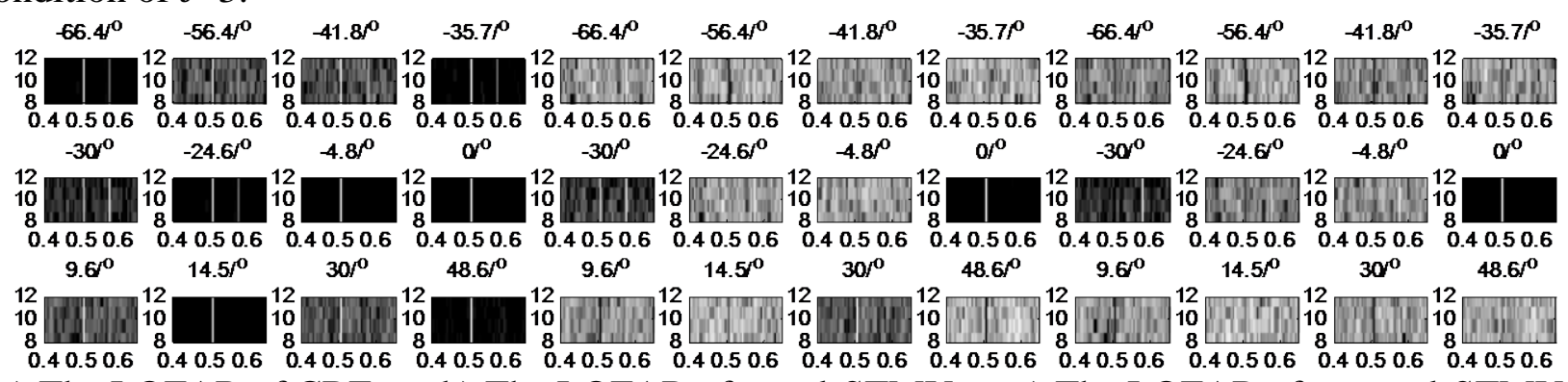
a) The LOFAR of CBF
b) The LOFAR of equal-STMV
c) The LOFAR of unequal-STMV

Fig 8 The LOFAR of multi-beamforming

In order to detect the weaker target a narrow beamwidth and a good control of the primary sidelobes of the strong target are important. Fig 8 shows that the tonal of Target A affected at all the other directions with CBF method. The equal-STMV can suppress the most of interference from adjacent direction except the high sidelobe tonals $\left(\gamma= \pm 30^{\circ}\right)$ emanated from $0^{\circ}$.However, the optimum coding STMV with unequal-STMV method could suppress all the rest interferences.

Fig 9 is the time-range in history pings. The x-axis is the detection period, and the y-axis is the ranger. The Fig a) is the history pings of mainlobe, and Fig b) is the history of high sidelobe emanated from mainlobe of strong target. The left is the CBF method, the right is the optimum coding STMV.

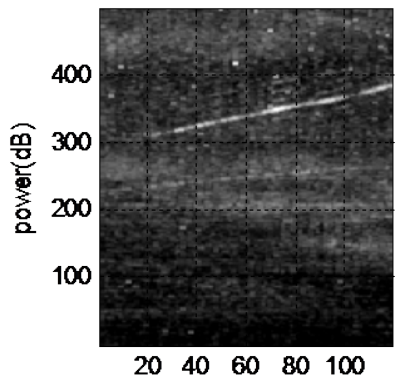

a) The history of mainlobe

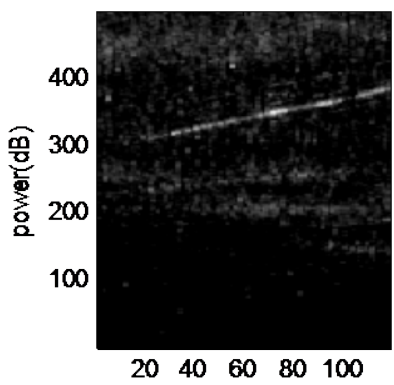

$20 \quad 40 \quad 60 \quad 80 \quad 100$

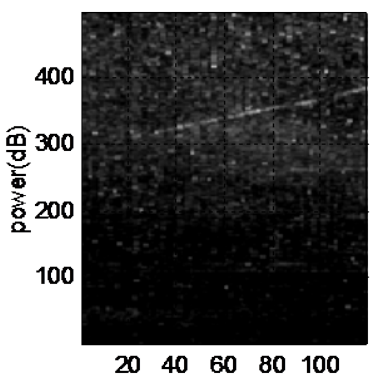

b) The history of sidelobe

The good performance of suppressing interferences is demonstrated by the trial data, which is performed both on the mainlobe and the sidelobe. We confirmed that the optimum coding STMV method have better performance. 


\section{Conclusions}

In this paper, we proposed optimum coding STMV beamformer which could solve the higer sidelobe of CBF with missing sensors, the proposed method could suppress the higer sidlobe and improve the reliable of detector .The simulation and the mathetical model and the sea trial proved the effective and feasibility.

\section{References}

[1] MaPeng-cheng, Li Dan and Yin Qin-Ye. A robust MVDR Beamforming Based on Covariance Matrix [J]. International Conference on Graphic and Image Processing, 2011.

[2] Francisco Rubio, Xavier Mestre, Walid Hachem. A CLT on the SINR of the Diagonally Loaded Capon/MVDR Beamformer [J]. 2011 IEEE International Conference on Acoustics, Speech and Signal Processing (ICASSP), 2011: 2644-2647.

[3] Ma Qi-ming, Wang Xuan-yin, and Du Shuan-ping. Research of the Method for the Weak Signal Detection Base on the Amplitude Fluctuation Property of the Frequency Spectrum[J]. Journal of Electronics \& Information Technology, 2008, 30(11): 2642-2645.

[4] Liu Kai, Liang Guo-long, and Ji Jian-fei. Noise Characteristics and Array Gain of An Acoustic Vector-sensor Array Influenced by Random Array Errors [J]. Journal of Harbin Engineering University, 2011, 32(5): 624-631.

[5] Chen Yang, Wang Zi-juan, and Zhu Dai-zhu. A Detecting Method for Line-spectrum Target Based on Variance-of -frequency Weight[J]. ACTA ACUSTICA, 2010, 35(1): 76-80.

[6] Shahriar Shirvani Moghaddam, Farida Akbari, Vahid Tabataba Vakili. A Novel Array Geometry to Improve DOA Estimation of Narrowband Sources at the Angles Close to the Array Endfire [J].2011 19th Iranian Conference on Electrical Engineering. 2011, May, 1-1.

[7] Jin Xue-lian, Yang Yi-xin, Wang Xiao-yu. Parallel Implementation of Wideband MVDR Beamforming on Multi-DSP for Sonar System [J].2011 IEEE International Conference on Signal Procssing, Communications and Computing. 2011, Sept, 1-4.

[8] Zhang Lei, Liu Wei, Yu Lei. Performance Analysis for Finite Sample MVDR Beamformer with Forward Backward Processing[J]. IEEE Transactions on Signal Processing, 2011, 58(5): 2427-2431.

[9] Wang Ping, Qian Bin. The Algorithm of Improving Detection performance with the Method of STAP Under the Reverberation Background[J]. Technical Acoustics, 2010, 29(6).

[10]Jin Yuan-wei, Benjamin. Reduced-Rank Adaptive Detection of Distributed Sources Using Subarrays [J]. IEEE Transactions on Signal Processing, 2005, 53(1): 13-25.

[11]Zhou Sheng-zeng. Research and Application of Fast-convergent Minimum Variance Distortionless Response Algorithm [J]. Chinese Journal of Acoustics, 2011, 30(3): 313-322.

[12]Yves Doisy, Laurent Deruaz, and Robert Been. Interference Suppression of Subarray Adaptive Beamforming in Presence of Sensor Dispersions [J]. IEEE Transactions on Signal Processing, 2010, 58(8): 4195-4212.

[13]D.N. Swingler. Steered Adaptive Subarray Beamforming [J]. Defense R\&D Canada Contractor Report Drea CR1999-089, July 1999.

[14]Mark Wilson, Ronald McHugh. A Comparison of Sparse and Periodic Arrays for Sonar [J].Proceedings of Eighth European Conference on Underwater Acoustics. 2006, June, 12-15.

[15]Han Hai-liang, Cheng Jian-qing, Yu Yun-zhi. Analysis on Effect of Amplitude-phase Errors for Performance of Sonar Uniform Linear Array [J]. Command control \& simulation, 2008, 30(2): 28-31.

[16]Wang Xin, Qian Bin. Unequally Spaced Linear Array Beamforming[J]. Technical Acoustics, 2010, 29(1): 90-93.

[17]Qian Bin,Wang Ping. Subarray Optimization and Partition Technology in Dimension Reduction Steered Minimum Variance[J]. Journal of Harbin Engineering University, 2013, 34(4) 